\title{
A New Algorithm for Ranging in Wireless Sensor Network
}

\author{
Kai Ruan ${ }^{1, \mathrm{a}}$, Xiaohui Chen ${ }^{1,2, \mathrm{~b}, *}$, Mengjiao Zhang ${ }^{1}$,Jiyuan Sun ${ }^{1}$, \\ Bangjun Lei ${ }^{1,2}$ \\ ${ }^{1 .}$ College of Computer and Information Technology, China Three Gorges \\ University, \\ 443002, Yichang, Hubei province, China \\ ${ }^{2}$ Collaborative Innovation Centre for Geo-Hazards and \\ Eco-Environment in Three Gorges Area, 443002, Yichang, Hubei \\ province, China. \\ *Corresponding author : Xiaohui Chen, chxh93@ctgu.edu.cn \\ ruankai88@126.com, ${ }^{b^{*}}$ chxh93@ctgu.edu.cn
}

\begin{abstract}
.
Traditional ranging model is not applicable due to the large ranging error under unknown environmental. In order to improve the ranging accuracy, this paper presents a new ranging model based on FUZZY - TS. The model analyzes the number of FUZZY rules and the parameters of the gaussian membership functions which impact on the model error. The simulation results have shown the superiority of the model on the nonlinear relationship between RSSI and d under the unknown environment. Thus it can be used in situations with little prior knowledge.
\end{abstract}

Keywords: Ranging;FUZZY-TS; WSN;RSSI

\section{Introduction}

With the development and needs of society, wireless sensor network (WSN) has been widely used in various field as a new information technology. Positioning, which is a prerequisite for many applications of WSN, is an important supporting technology in WSN [1]. Currently there are many localization algorithms have been proposed. They can be divided into two categories[2]: ranging-based and ranging-free positioning algorithms. The ranging-based methods exist four different ways, which are respectively based on time difference of signal arrival (TDOA), angle of arrival (AOA), time of signal arrival (TOA) and receiving signal strength indication (RSSI) [3]. The method based on RSSI is low power consumption, low cost, and simple,so the 
paper choose it to study.

Some researchers propose an improved equation for distance estimate based on RSSI [4], the method adds random error in the classical ranging model. The method of multiple measurements has been proposed to eliminate the impact of the environment through the way of weighted and average [5], then the distance is calculated by the classical ranging-based model. In paper [6], the calibration algorithm of Gaussian model has been added in the RSSI measurement, it eliminates the path dissipation index. The paper [7] proposes a hybrid filtering and least squares ranging method based dynamic estimation of the environments parameters. The paper [8] proposes an amendment for the environment parameters based on the least squares, the method correct the environmental parameters through the fitting of least squares to eliminate the effects of various disturbances. A hybrid positioning algorithm which combines the RSSI and TDOA has been proposed [9]. In this paper, we apply the TS model to fit the relationship between the RSSI and distance. A new ranging algorithm based on FUZZY-TS has been proposed. The method is self-adaptive in complex environment and improves the ranging accuracy to improve the positioning accuracy.

\section{Classical Ranging Model}

The traditional Received Signal Strength Indicator(RSSI) logarithmic model can be showed in the following equation:

$$
\begin{gathered}
R S S I(d)=A-n \lg (d) \\
d=10^{\frac{A-R S S I}{n}}
\end{gathered}
$$

Where,RSSI is the received signal strength ; $\mathrm{n}$ indicates the path loss exponent which is relative with the environment; $A$ is the unit of distance of received signal strength.

Fig.1 depicts the different $\mathrm{A}$ and $\mathrm{n}$ for the RSSI and the $\mathrm{d}$ relation in the model.

In the non-free space, due to the complex of environment and interference, it is prone to lead reflection, diffraction, refraction and scattering of the wireless signal and other interfering factors. So we couldn't use the unified parameter model to present the relationship between RSSI and d. In this paper, we divide the entire interval into three sections to simulate the relationship between RSSI and distance . Fig.2 shows the curve of between the RSSI and d under assuming environment. 


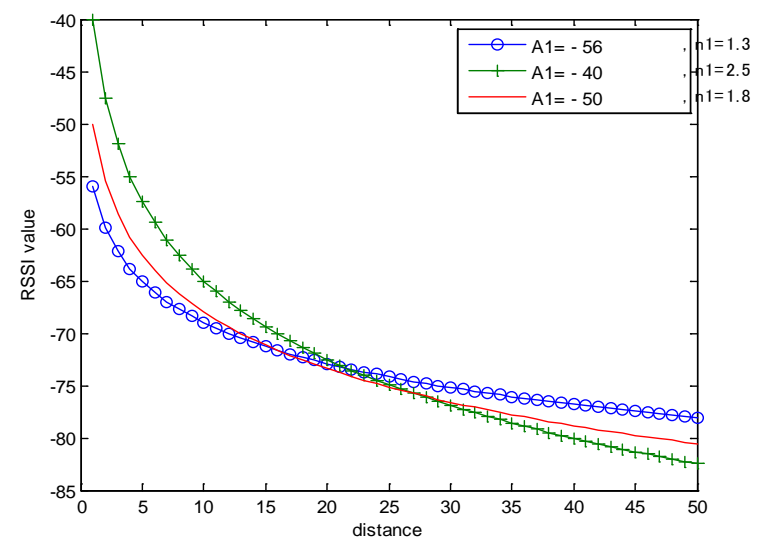

Figure1. Curves of classical ranging model with different parameters

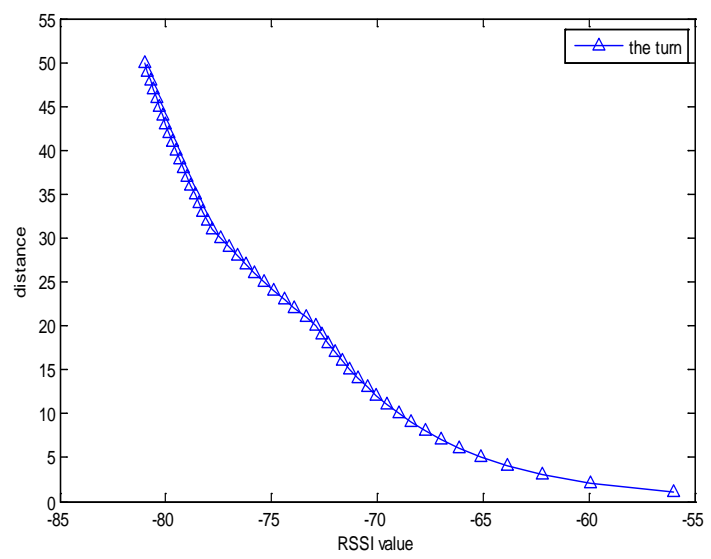

Figure2. Curve of RSSI and d under simulation environment

\section{The Establishment of the Fuzzy TS Range Model}

In this paper, the algorithm design steps are as follows:

(a) Using $\mathrm{n}$ fuzzy rules to fit the nonlinear relationship between RSSI and distance, The ith fuzzy rule can be described by the IF-THEN language as follows:

$$
\text { if } \mathrm{R} \text { is } \mathrm{A}^{\mathrm{i}} \text { Then } \mathrm{d}^{i}=P_{0}^{i}+P_{1}^{i} \mathrm{R}
$$

(b) Total output of the system is:

$$
\mathrm{d}=\left(\mu^{1} d^{1}+\mu^{2} d^{2}+\cdots+\mu^{n} d^{n}\right) / \sum_{i=1}^{n} \mu^{i}
$$

Where $\mu^{\mathrm{i}}$ is the membership degree of each rule.In this paper, it using a 
Gaussian membership function.

(c) Putting $\mathrm{d}^{\mathrm{i}}$ into the total output of the system , then there is:

$$
\begin{aligned}
& \text { where } \quad \begin{array}{l}
\mathrm{d}=H \bullet \theta^{T} \\
\left.\theta=\left(P_{0}^{1}, P_{1}^{1}, P_{0}^{2}, P_{1}^{2}, \cdots P_{0}^{n}, P_{1}^{n}\right)^{T} \quad w^{2}, w^{2} \mathrm{R}, \cdots, w^{n}, w^{n} \mathrm{R}\right)
\end{array} \\
& \theta \mu_{i=1}^{i} \mu^{i},
\end{aligned}
$$

By the least squares method, obtaining the optimal conclusion parameters of the minimum squared error sense, which is calculated as follows:

(d) Calculated:

$$
\begin{aligned}
& F_{k}=S_{k-1} H_{k}{ }^{T} /\left(1+H_{k} S_{k-1} H_{k}{ }^{T}\right) \\
& S_{k}=S_{k-1}-F_{k} H_{k} S_{k-1} \\
& \theta_{k}=\theta_{k-1}+F_{k}\left(y_{k}-H_{k} \theta_{k-1}{ }^{T}\right)
\end{aligned}
$$

Where $S_{k}$ is covariance matrix, $F_{k}$ is gain vector, $\theta_{k}$ is parameter vector to be identified, $\mathrm{H}_{\mathrm{k}}$ is row vector .

(e) $k=k+1$, if $k \leq L$, then return (d), where $\mathrm{L}$ is the number of samples. After the iteration is completed, $\theta_{\mathrm{k}}$ is the best Conclusion parameters.

\section{Simulation and Analysis}

In the surroundings of the Fig.2,we set the different number of fuzzy rules to build the FUZZY-TS ranging model to learn it. The follow picture is ranging model of the different number of fuzzy rules and the curve under the simulation environment.

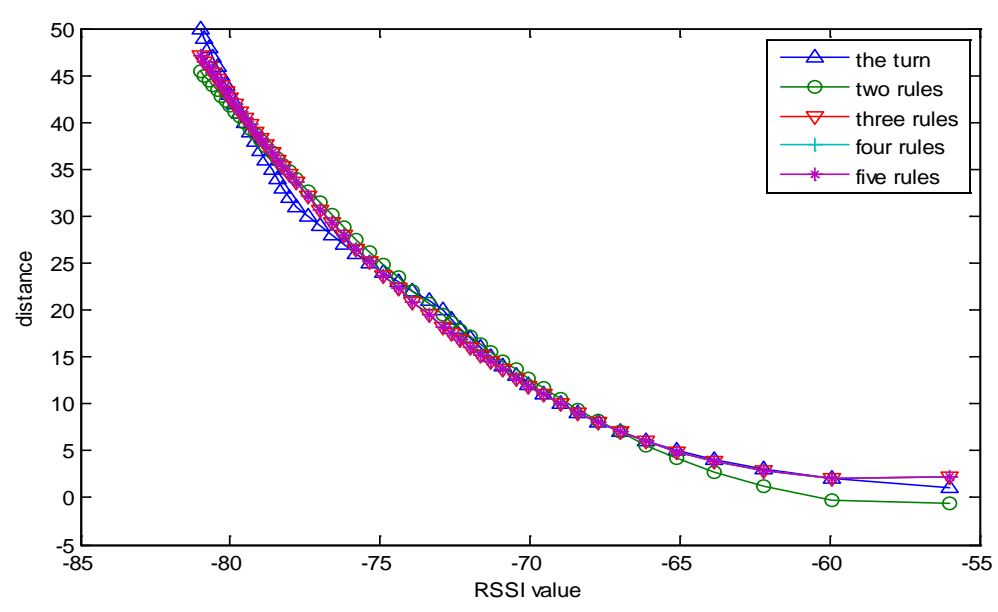

Figure3.The ranging model curve of the different number of fuzzy rules 
Table.1 The Average Errors Of The Different Number Of Fuzzy Rules

\begin{tabular}{|c|c|c|c|c|}
\hline FUZZY-TS Model & 2 rules & 3 rules & 4 rules & 5 rules \\
\hline average error & 0.1146 & 0.0602 & 0.0487 & 0.0472 \\
\hline
\end{tabular}

According to the Fig.3 and the above table.1, we know the FUZZY-TS ranging model can fitting nonlinear relationship of RSSI and distance very well. When the number of fuzzy rules is more, the error of the model is the least.But we select more the number of fuzzy, we find that the calculation is very complicated and the error of the model reduces very less. On account of the different environment, we need to determine rational number of fuzzy rules. In this paper, we select 3 fuzzy rules.

This paper uses the Gaussian membership function $\mu(\mathrm{R}, \sigma, u)=e^{-\left(\frac{\mathrm{R}-u}{\sqrt{2} \sigma}\right)^{2}}$. where $\mu$ refers to the fuzzy center ,o refers to the Fuzzy radius. Then we discuss the different $\mu$ and o on the error influence of the FUZZY - TS ranging model. This simulation uses three fuzzy rules, so it needs three membership degrees.

When the fuzzy radius of the three membership functions are selected the same,Then it changes the fuzzy center $\mu$. Fig.4 shows the curve of Ranging model error of the the different fuzzy centers.

When fixing the fuzzy center of the three membership functions.And changing the size of the fuzzy radius o. Fig.5 show the curve of Ranging model error of the the different fuzzy radius.

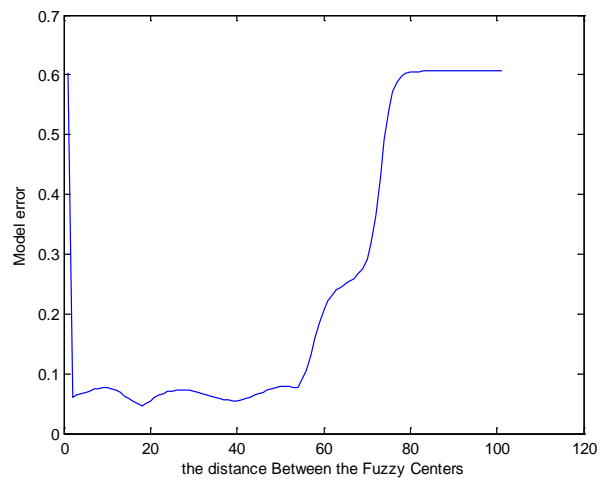

Figure4. Ranging model error of the the different fuzzy centers 


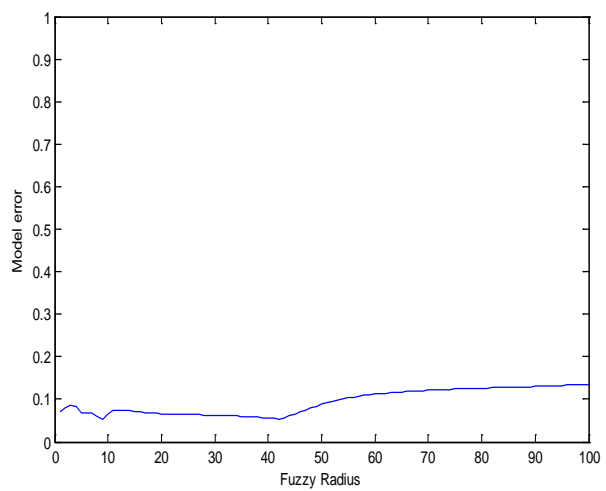

Figure5. Ranging model error of the the different fuzzy radius

From the fig.4, the error of the model is small and has less volatility, when the distance $d$ in $3-50$ or so. When $d$ is greater than 50 , the error of the model is growing. Through analyzing, when $\mathrm{d}$ is small, the three membership functions almost overlap. When $d$ is larger, some data belong to the membership degree is the relatively small,and its value can be regarded as 0 . So it cannot be very good to learn in this model .

From the fig.5, we can find that the FUZZY - TS model error has a little change with the increase of the fuzzy radius in the certain range. When or is relatively large, the error of the model increases slowly.

\section{Conclusion}

This paper analyzes the classic ranging model.The impact of the environment differs as the distance increases, therefore the classic ranging model cannot meet the requirements. This paper presents the FUZZY - TS ranging model which has the adaptive learning ability. The result of simulation shows that the model can very good to fit the RSSI and d of the non-linear relationship in the unknown environment. At the same time, the paper also analyzes the influence of the different number of the fuzzy rules and the parameters of the membership degree in the model. The simulation results show that the model can be used in the parameters of the unknown environment, thus it can be applied to the unknown environment.

\section{Acknowledgement}

This project is supported by 2012 Natural Science Foundation of Hubei Province (2012FFC09701).

This project is supported by NSFC project "Feature pool driven based cluster tracking under complex environment (61102155)” . 


\section{References}

[1]Peng Yu ,Wang Dan .A review: wireless sensor networks localization[J]. JOURNAL OF ELECTRONIC MEASUREMENT AND INSTRUMENT ,2011,05:389-399.

[2]He T, Huang C, Blum B M, et al. Range-free localization schemes for large scale sensor networks[C]//Proceedings of the 9th annual international conference on Mobile computing and networking. ACM, 2003: 81-95.

[3] Demirbas M, Song Y. An RSSI-based scheme for sybil attack detection in wireless sensor networks[C]//Proceedings of the 2006 International Symposium on on World of Wireless, Mobile and Multimedia Networks. IEEE Computer Society, 2006: 564-570.

[4]Awad A, Frunzke T, Dressler F. Adaptive distance estimation and localization in WSN using RSSI measures[C]//Digital System Design Architectures, Methods and Tools, 2007. DSD 2007. 10th Euromicro Conference on. IEEE, 2007: 471-478.

[5]WANG Wei,CHEN Dai, ZHOU Yong . RSSI localization algorithm based on distance and position correction [J].Computer Engineering and Design,2011,02:409-412+622.

[6]FANG Zhen,ZHAO Zhan,GUO Peng,ZHANG Yuguo. Analysis of Distance Measurement Based on RSSI[J]. CHINESE JOURNAL OF SENSORS AND ACTUATORS,2007,11:2526-2530.

[7]WAN Guofeng,ZHONG Jun,YANG Chenghui. Improved algorithm of ranging and locating based on RSSI[J]. Application Research of Computers,2012,11:4156-4158.

[8]TAO Weige,ZHU Yihua,JIA Ziyan. A Distance Measurement Algorithm Based on RSSI Hybrid Filter and Least Square Estimation [J].CHINESE JOURNAL OF SENSORS AND ACTUATORS ,2012,12:1748-1753.

[9]DU YaJiang,GAO LiBing. RSSI Parameter Calibration Method Based on the Least Square Algorithm[J].COMPUTER SYSTEMS

\&APPLICATIONS ,2012,02:221-224. 